\title{
MULTISCALE COMPOSITE 3D FINITE ELEMENT FOR LUNG MECHANICS
}

\author{
M. Kojic ${ }^{1,2,3}$
}

${ }^{1}$ Bioengineering Research and Development Center BioIRC Kragujevac, 6 Prvoslava Stojanovića Street, 3400 Kragujevac, Serbia

${ }^{2}$ Houston Methodist Research Institute, The Department of Nanomedicine, 6670 Bertner Ave., R7 117, Houston, TX 77030, USA

${ }^{3}$ Serbian Academy of Sciences and Arts, 35 Knez Mihailova Street, 11000 Belgrade, Serbia e-mail: mkojic42@gmail.com

\begin{abstract}
The lungs are the pair of organs where very complex internal microstructure provides gas exchange as the vital process of living organisms. This exchange in humans occurs within only $300 \mathrm{~g}$ of tissue but on the surface of millions of alveoli with the total surface area of around $130 \mathrm{~m}^{2}$. The extremely complex microstructure consists of micron-size interconnected channels and alveoli, which significantly change the size during breathing and remain open. These conditions are maintained due to existence of two mechanical systems - one external and the other internal, which act in the opposite sense, so that the lung behaves as a tensegrity system. Many computational models, with various degrees of simplifications and sophistication have been introduced. However, this task remains a challenge. We here introduce a 3D multi-scale composite FE for mechanics of lung tissue (MSCL). The model can be further used in generating computational models for mechanics for the entire lung and coupling to airflow.
\end{abstract}

Keywords: mechanics of lung microstructure, multi-scale 3D composite finite element, lung tissue material models, surfactant.

\section{Introduction}

Investigation of lung function, which includes airflow, gas exchange and mechanics of deformation, has been the subject of experimental and theoretical investigations over centuries. Different concepts of formulating the background of how the lung functions have been introduced. We first cite Ewald R. Weibel, one of the leading authorities regarding the lung science (Weibel 2013): "The lung is faced with a particularly difficult design problem: to maintain an alveolar surface area of $130 \mathrm{~m}^{2}$ suspended in an air space of a few liters with only about 300 $\mathrm{g}$ of tissue, and this against surface forces acting on the complex air-tissue interface. The air volume must be variable according to ventilatory demands so that the support structures must be made of soft tissue, primarily connective tissue fibers". The main process of gas exchange occurs within the lung parenchyma, which is composed of small airways called bronchioles and ductalveolar system. During breathing, the lung microstructure deforms significantly providing airflow in a cycling manner. It is practically impossible to represent the complex microstructural 
geometry accurately in a mathematical model for computing either mechanics of deformation or airflow within large domains of the lung. A significant number of models have been introduced for description of the lung mechanics for elucidating the lung function. We cite several of these that are the most relevant for our formulation of a multi-scale finite element.

We start with the seminal works (Weibel 1963) and (Weibel and Gil 1977) where they introduced a fundamental concept of the loading carrying system of the lung. The basic microstructural unit is represented by a duct, shown in Fig. 1a. Further, the model has been specified in (Wilson and Bachofen 1982) in a way shown in Fig. 1b. There exist systems of two groups of fibers - here called external and internal fibers, which are carrying the mechanical forces within the lung; these fibers are called by (Weibel 2013) as peripheral and axial fibers.



a

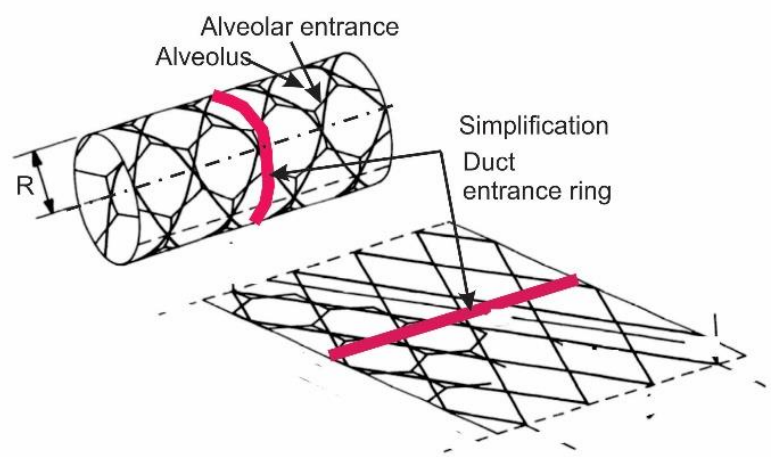

b

Fig. 1. Microstructural model of Weibel. a) Scanning electron micrographs of a rabbit lung duct and alveoli with forces acting on the internal fibers. b) Fibers and alveoli in the duct, according to (Wilson and Bachofen 1982), and the duct entrance ring as simplification used in our model.

The internal system of fibers serves to keep the alveoli open so that the entire microstructure behaves as a 'tensegrity' structure (Fuller 1961, Ingber 2003). Surfactant, covering the microstructural tissue, also plays important role for the lung functioning, which will further be taken into account in our models. Another duct model, important for the insight into forces within microstructure, is shown in Fig. 2. A schematic of duct model is displayed in Fig. 2a, while forces acting in the model are shown in Fig. 2b, according to (Greaves et al. 2010). It is important to notice that the forces produced by deformation of external tissue and surfactant ( $F_{A t}^{e x t}$ and $F_{A \gamma}^{e x t}$ ), and by alveolar septa and surfactant $\left(F_{B t}^{\mathrm{int}}\right.$ and $\left.F_{B \gamma}^{\mathrm{int}}\right)$, are acting in parallel. On the other hand, $F_{B t}^{\mathrm{int}}$ and $F_{B t}^{\mathrm{int}}$ forces, and force in the tissue of rings $\left(F_{C t}^{\mathrm{int}}\right)$, act as forces in series. Practically, forces $\left(F_{B t}^{\mathrm{int}}\right.$ and $\left.F_{B \gamma}^{\mathrm{int}}\right)$ vs. are in balance, stretching the alveolar septa tissue. 


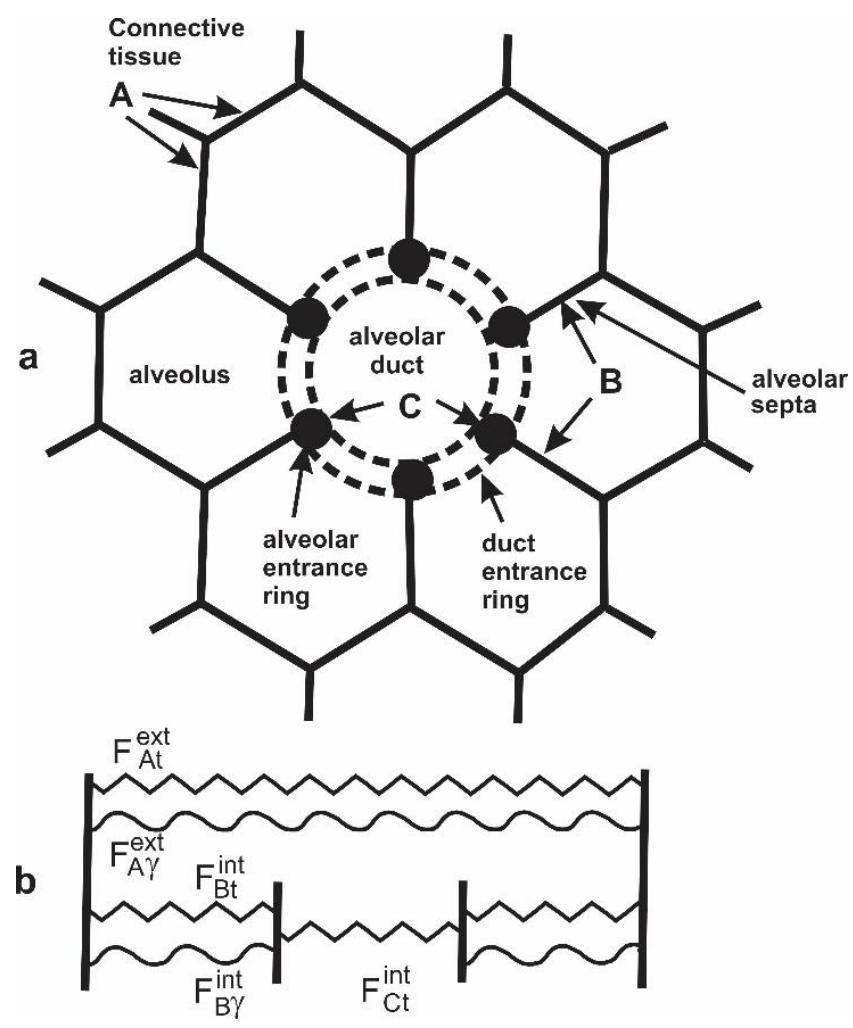

Fig. 2. Alveolar duct model according to Greaves et al. (2010). a) Schematic of the duct structure which includes external tissue (A) connected to alveoli, with alveolar septa (B) and entrance rings, and duct entrance ring $(\mathrm{C})$; b) Forces generated by structural deformation: $F_{A t}^{\text {ext }}$ and $F_{A \gamma}^{e x t}$ - external connective tissue and surfactant, $F_{B t}^{\mathrm{int}}$ and $F_{B \gamma}^{\mathrm{int}}$ - alveolar tissue and surfactant, and $F_{C t}^{\text {int }}$ - alveolar and entrance rings.

Deformation of the lung internal geometry induces change of the volume of the air space and air pressure distribution within the lung, which altogether produce the airflow deep in the lung. The airflow is conditioned by the microstructure deformation, and coupling mechanical deformation with airflow is the ultimate goal of modeling a lung. We further give a brief overview of references related to airflow. Flow patterns are very complex due to the complexity of lung microstructure (complexity of lung microstructure can be seen in almost all publications related to lung, e.g. (Tsuda et al. 2008, Y. Fukuoka et al. 2014) inducing air mixing and gas exchange, as well as chaotic transport of small particles and molecules. These aspects of airflow have been studied over decades, as in (Tsuda et al. 1995, 2011, Henry et al. 2002, Miki et al. 1993, Kojic and Tsuda 2004, Kojic et al. 2011). Modeling of airflow within the lung remains a challenge. It is practically impossible to model in all details airflow in the large domains of the lung; therefore, simple models that capture main flow characteristics are desired. Finally, coupling mechanics of lung deformation and airflow represents an important and extremely challenging task due to overall complexity described in, for example, (Liu et al. 1998) outlined above. 


\section{Formulation of a 3D multi-scale composite finite element for mechanics of lung}

Based on the above analysis of the micromechanical models, we formulate a duct model as shown in Fig. 3, considering, as the other authors, the duct as a fundamental microstructural unit.

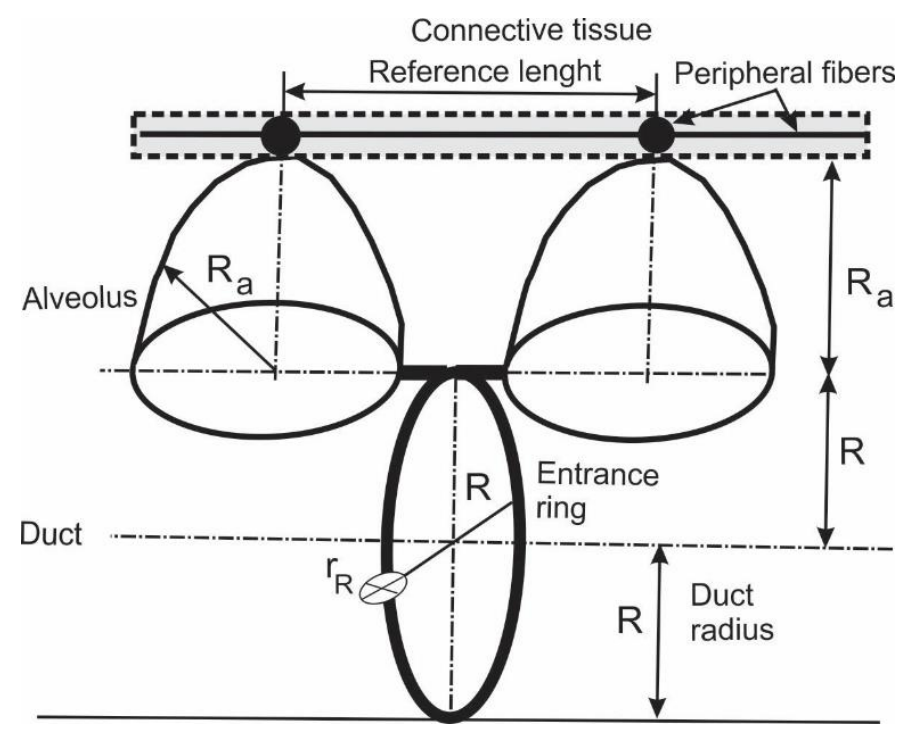

Fig. 3. Our duct model as the basic unit of the lung microstructure. Consists of entrance rings of the radius $R$ and cross-sectional radius $r_{R}$, and alveoli (semi-spheres of radius $R_{a}$ ) covered by surfactant. The duct is connected to peripheral (external) radial and axial fibers of the connective tissue.

The model description is as follows. We consider the duct as a cylinder of the radius $R$, where the internal fiber network is represented by duct entrance rings (called further entrance rings) of certain representative cross-sections (the radius $r_{R}$ in the figure) at the distances specified as the reference lengths. The rings are connected to alveolar rings which support the alveolar septa. In the model we will neglect the stiffness of alveolar rings (but they can be added in a straightforward way) and only take into account stiffness of the alveolar septa. The outer duct radius is equal to $R+R_{a}$, where $R_{a}$ is the alveolar radius, with considering alveoli as semi-spheres. We assume that there is a number of alveoli in the circumferential direction of duct corresponding to one entrance ring, specified by a number $N_{R}$. The alveoli are covered by surfactant at both surfaces, with the surface tension $\gamma$. The alveoli are connected to the external fibers (radial and axial) of the connective tissue; this tissue has a cylindrical surface of the radius $R+R_{a}$. It assumed that the external connective tissue surface is also covered by surfactant. The length of the duct is $L_{d u c t}$ and the axial alveolar density, as number of alveoli per unit duct length), is $n_{a}$. This density is also equal to $N / L_{\text {duct }}$ where $N$ is number of the entrance rings.

The internal structure deforms due to deformation of the external tissue which carries the external load of the lung and is specified by the stretch $\lambda$, which, in formulating the model, is considered known. The external tissue is also loaded by the forces coming from the internal microstructure deformation, transferred by alveolar septa (as can be seen from Figs. 1 and 2). On the other hand, the forces coming from alveolar septa deformation and surfactant are in balance with forces from entrance rings. In order to determine how duct microstructure deforms for a given deformation of lung as a continuum (on macroscopic scale) we calculate the stretch at a given continuum point as 


$$
\lambda=1+\left(e_{x x}+e_{y y}+e_{z z}\right) / 3=1+e_{V}
$$

where $e_{x x}, e_{y y}, e_{z z}$ are strains and $e_{v}$ is the mean volumetric strain, calculated from the displacement field in a FE model. Then the virtual work of the mechanical system corresponding to one entrance ring is equal to zero, since the system is equilibrium

$$
\begin{aligned}
\delta W=\delta W_{a}+\delta W_{\text {surf }}+\delta W_{R}=2 N_{R} \int\left(\sigma_{\text {Va }}(\right. & \left.+\frac{2 \gamma}{\delta_{a}}\right) \frac{\delta R_{a}}{R_{a}} d V_{a}+ \\
& +\int_{V_{\text {Ring }}}\left[(1-m) \sigma_{c t}+m \sigma_{m}\right] \frac{\delta R}{R} d V_{\text {Ring }}=0
\end{aligned}
$$

where (besides the above defined) $m$ is ratio of muscle cells volume within the ring with respect to total volume; $\sigma_{a}$ is the stress in alveolar septa; $\sigma_{c t}$ and $\sigma_{m}$ are stresses in connective and muscle tissue of the ring, since the ring is composed of these tissue types. Stresses and surfactant are assumed to be constant over volumes and surface of alveoli; and stresses in ring are assumed uniform. It is used in (2) that the stress in the alveolar wall can be expressed as

$$
\sigma_{\gamma}=\gamma / \delta_{a}
$$

and that the strain variation within alveolus and ring are

$$
\delta e_{a}=\frac{\delta R_{a}}{R_{a}} \text { and } \delta e_{\text {Ring }}=\frac{\delta R}{R}
$$

In addition, it is taken that stresses and strains in any two orthogonal directions within the surface of alveoli are the same, i.e. we have

$$
\sigma_{x x}=\sigma_{x x}=\sigma_{a}, \quad \delta e_{x x}=\delta e_{y y}=\frac{\delta R_{a}}{R_{a}}
$$

The volumes of alveolus and ring are

$$
V_{a}=4 \pi R_{a}^{2} \delta_{a}, \quad V_{\text {Ring }}=2 \pi A_{\text {ring }} R
$$

Also, we have that

$$
R+R_{a}=\lambda\left(R_{0}+R_{a 0}\right)
$$

and

$$
\delta R_{a}=-\delta R
$$

Substituting the above relations into (2), we obtain the balance equation as

$$
4 N_{R}\left(\delta_{a} \sigma_{a}+2 \gamma\right) R_{a}-\left[(1-m) \sigma_{c t}+m \sigma_{m}\right] A_{\text {ring }}=0
$$

This equation can be reduced to one unknown, say the radius $R$, if we include constitutive relations for stresses and surface tension. Namely, we have that the stress within alveolar tissue depends on the stretch $\lambda_{a}$ within alveolus, 


$$
\begin{aligned}
& \lambda_{a}=\frac{R_{a}}{R_{a 0}} \\
& \sigma_{a}=\sigma_{a}\left(\lambda_{a}\right)=\sigma_{a}\left(\frac{R_{a}}{R_{a 0}}\right)
\end{aligned}
$$

and surface tension is a function of the alveolar area change $\lambda_{\gamma}$,

$$
\begin{gathered}
\lambda_{\gamma}=\frac{R_{a}^{2}}{R_{a 0}^{2}} \\
\gamma=\gamma\left(\frac{R_{a}^{2}}{R_{a 0}^{2}}\right)
\end{gathered}
$$

The stresses within the ring depend on the stretch within the ring,

$$
\lambda_{R}=\frac{R}{R_{0}}
$$

i.e.

$$
\sigma_{c t}=\sigma_{c t}\left(\lambda_{R}\right), \sigma_{m}=\sigma_{m}\left(\lambda_{R}\right)
$$

The governing equation (9) is a nonlinear and must be solved numerically, for example, by a bisection scheme with an acceleration scheme as in (Kojic and Bathe 2005), or within the computer program PAK (Kojic at al. 2008). The computations scheme, with $R$ as variable, would be: assume $R$, calculate the radius $R_{a}$ from (7), stretches $\lambda_{a}$ from (10), $\lambda_{\gamma}$ from (11) and $\lambda_{R}$ from (13); then determine stresses $\sigma_{a}, \sigma_{c t}, \sigma_{m}$ and surface tension $\gamma$ from constitutive laws; iterate on $R$ until the equation (9) is satisfied within a selected error tolerance.

After the solution of equation (9) is obtained, the stresses acting on surface of the external tissue of the duct can be calculated. The normal stress on the cylindrical surface, of the radius $R+R_{a}$ and length $L_{d u c t}$, balances the force produced by $N$ rings, hence we have

$$
\sigma_{\text {tiss }}^{\gamma}=\frac{2\left[(1-m) \sigma_{c t}+m \sigma_{m}\right] A_{\text {ring }}}{R+R_{a}} \frac{N}{L_{\text {duct }}}=\frac{2 n_{a}\left[(1-m) \sigma_{c t}+m \sigma_{m}\right] A_{\text {ring }}}{R+R_{a}}
$$

Besides these stresses, surfactant covering the external duct surface has the mechanical effects. We adopt the condition that the surface has the same deformations in radial and axial directions, so that they are equal to the mean volumetric strain $e_{V}$ of the continuum (equation (1)),

$$
e_{\text {rad }}=e_{\text {axial }}=e_{A}=e_{V}
$$

The virtual work of surfactant on the external surface, for the entire duct, is

$$
\delta W_{\text {ext }}=\gamma \int_{A}\left(\delta e_{\text {rad }}+\delta e_{\text {axial }}\right) d A=2 \gamma A \delta e_{A}=2 \pi \gamma\left(R_{a}+R\right) L_{\text {duct }} \delta e_{A}
$$

Virtual work per unit volume of air of the duct is 


$$
\delta \bar{W}_{\text {air }}=\frac{2 \pi \gamma\left(R_{a}+R\right) L_{d u c t} \delta e_{A}}{\left(R^{2} \pi+2 / 3 \pi R_{a}^{3} n_{a}\right) L_{d u c t}}=\frac{2 \gamma\left(R_{a}+R\right)}{R^{2}+2 / 3 R_{a}^{3} n_{a}} \delta e_{A}
$$

Virtual work per unit elementary volume of tissue is

$$
\delta \bar{W}_{\text {tissue }}=\frac{1-r_{V a}}{r_{V a}} \delta \bar{W}_{\text {air }}=\frac{1-r_{V a}}{r_{V a}} \frac{2 \gamma\left(R_{a}+R\right)}{3\left(R^{2}+2 / 3 R_{a}^{3} n_{a}\right)} \delta e_{V}=\sigma_{e x t}^{\gamma} \delta e_{V}
$$

where

$$
\sigma_{e x t}^{\gamma}=\frac{1-r_{V a}}{r_{V a}} \frac{2 \gamma\left(R_{a}+R\right)}{3\left(R^{2}+2 / 3 R_{a}^{3} n_{a}\right)}
$$

is the stress within tissue corresponding to action of surfactant, and $r_{V a}$ is volumetric fraction of air.

Finally, we here give the incremental-iterative equations of linear momentum balance for the MSCL finite element which include the stresses due to microstructural deformation. For a time step $\Delta \mathrm{t}$ and iteration ' $i$ ', they are (Kojic et al. 2008)

$$
\left(\frac{1}{\Delta t} M_{I J}+K_{I J}\right)^{(i-1)} \Delta V^{J(i)}=F_{I}^{e x t(i)}+F_{I}^{\gamma(i-1)}-\left(\frac{1}{\Delta t} M_{I J}+K_{I J}\right)^{(i-1)} V^{J(i-1)}+\frac{1}{\Delta t} M_{I J}^{(i-1)} V^{t J}
$$

where

$$
\begin{aligned}
& M_{I J}=\int_{V}\left(1-r_{V a}\right) N_{I} N_{J} d V \\
& K_{I J}=\int_{V}\left(1-r_{V a}\right) B_{I i} C_{i j} B_{j J} d V \\
& F_{I}^{\gamma}=\int_{V} B_{I i}\left(\sigma_{\text {tiss }}^{\gamma}+\sigma_{\text {ext }}^{\gamma}\right)_{i} d V
\end{aligned}
$$

The detailed description of the above matrices is given elsewhere (e.g. Kojic et al. 2008). The constitutive matrix $C_{i j}$ includes characteristics of external connective tissue and constitutive relations of the microstructure. Note that the volumetric fraction of the air changes during deformation. It can be calculated as

$$
r_{V a}=\eta r_{V a 0}
$$

where $r_{V a 0}$ is the initial value, and coefficient $\eta$ is

$$
\eta=\frac{\pi\left(R+R_{a}\right)^{2} L_{d u c t}}{\pi\left(R_{0}+R_{a 0}\right)^{2} L_{d u c t 0}}=\lambda \frac{\left(R+R_{a}\right)^{2}}{\left(R_{0}+R_{a 0}\right)^{2}}
$$

\section{Numerical example - multi-scale composite 3D finite element of lung microstructure under uniform extension}

Here, a simple example to illustrate the main features of the formulated MSCL finite element is presented. 
A 3D element, shown in Fig. 4a, is subjected to the same extension in all three directions.

a

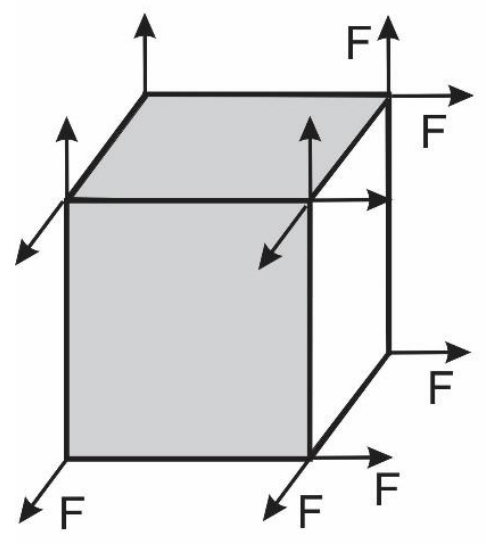

b

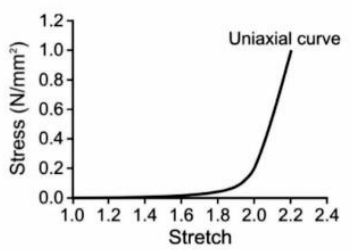

1)

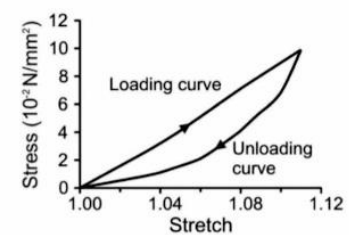

3)

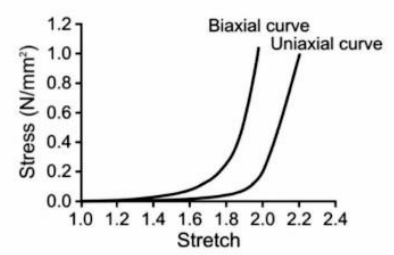

2)

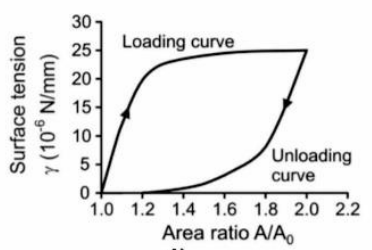

4)

Fig. 4. Uniform extension of a 3D material element representing lung continuum. a) Material element of unit size $(1 \mathrm{~mm})$ is loaded in all directions by a forces F. b) Constitutive curves for microstructure according to (Kojic et al. 2006, 2011) : b-1) Uniaxial curve for connective tissue, (Fukaya, et al. 1968); b-2) Biaxial curves for alveolar septa, (Fukaya, et al. 1968, Hildebrandt et al. 1969); b-3) Hysteretic characteristic of muscle tissue within entrance ring, (Sasaki and Hoppin 1979); b-4) Surfactant hysteresis, (Wilson 1982).

Data for the microstructure are taken as follows (lengths in $\mathrm{mm}$ ):

Geometrical data

Duct radius- $\mathrm{R}=0.1$, alveolar radius $\mathrm{R}_{\mathrm{a}}=0.3$, alveolar thickness $\delta_{a}=0.05$

Radius of entrance ring cross-section $r_{\text {ring }}=0.4$, muscle content $\mathrm{m}=0.5$

Radial alveolar density $\mathrm{N}_{\mathrm{R}}=5$, axial alveolar density $\mathrm{n}_{\mathrm{a}}=1 /\left(\mathrm{R}_{\mathrm{a}}\right)=1.667$

Material data

Connective tissue - external and within entrance ring - uniaxial curve, Fig. 5b-1

Alveolar septa - biaxial curve, Fig. 5b-2

Muscle tissue within entrance ring - hysteretic curve, Fig. 5b-3

Surfactant over alveoli and external tissue surface - hysteretic curve, Fig. 5b-4

Volumetric fraction of air $\mathrm{r}_{\mathrm{Va}}=0.5$. Force increases linearly to maximum $\mathrm{F}=3.50 \mathrm{e}-03 \mathrm{~N}$ and decreases back to zero.

The volume change (in $\mathrm{mm}^{3}$ ) in terms of stress within the external connective tissue is shown in Fig. 5. It displays the hysteretic response of the MSCL finite element. Stresses are rather small since the constitutive curve for connective tissue corresponds to the soft tissue represented by the constitutive curve in Fig. 5b-1. It can be seen the hysteretic characteristic of the formulated MSCL finite element, what was the main goal of this example. 


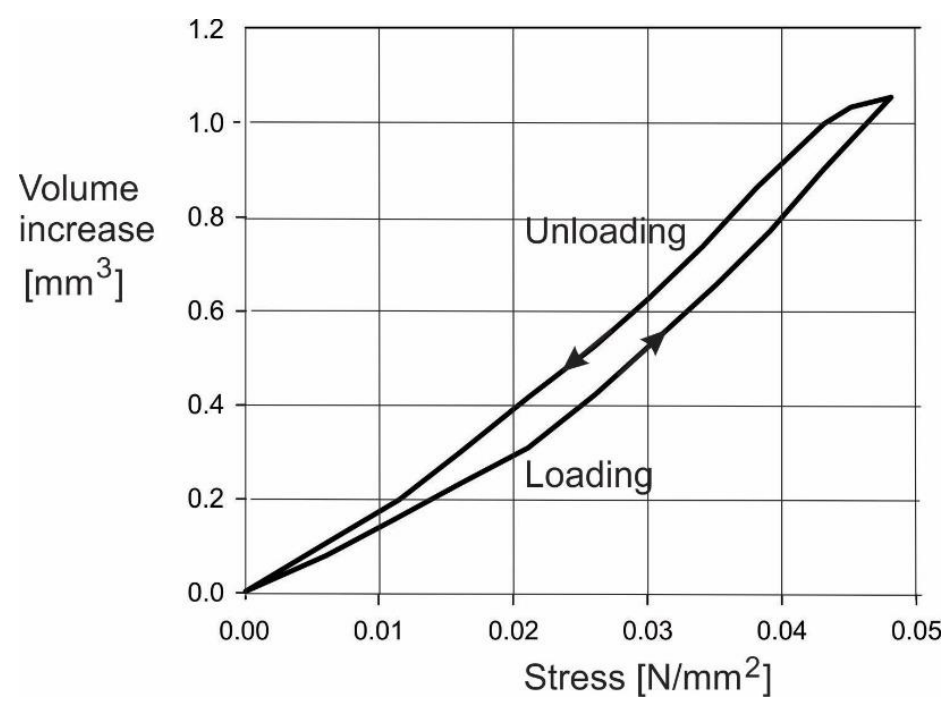

Fig. 5. Hysteretic response of the MSCL element under volumetric loading and unloading. Volume increase vs. stress within external connective tissue. The maximum volume is more than doubled with respect to the initial, which is $1 \mathrm{~mm}^{3}$.

\section{Conclusions}

The introduced MSCL finite element for lung mechanics includes characteristics of the lung macroscale continuum medium, represented as connective tissue, and mechanical properties of the microstructure. It is designed for modeling mechanics of the lung in a simple manner by 3D finite elements. The external load of the lung caused by transmural pressure on the lung surface is balanced by the stresses within the connective tissue. This tissue is also stretched by the stresses produced by the internal microstructural deformation of alveolar septa and internal fibrous structure (represented by entrance ductal rings) and by surfactant covering the microstructural surfaces.

The MSCL finite element incorporates change of the shape and size of the microstructural geometry and provides a basis for a direct coupling with the airflow within the lung. This straightforward coupling can be used for modeling the entire lung in a way feasible for numerical simulation of various problems of the lung, spanning from airflow to drug delivery in a healthy lung as well as in a lung with different abnormalities and diseases, including tumors.

\section{Acknowledgements}

The author acknowledges support from the City of Kragujevac, Serbia, Ministry of Education and Science of Serbia (grants OI 174028 and III 41007) and from National Cancer Institute under grant U54 CA210187. This work is supported by the SILICOFCM project that has received funding from the European Union's Horizon 2020 research and innovation programme under grant agreement No 777204.

The author is thankful to Dr. Akira Tsuda, Harvard School of Public Health, (retired), for very useful discussion and suggestions during this work. 


\section{References}

Fukaya, H., Martin, C. J., Young, A. C., and Katsura, S. (1968), Mechanical Properties of Alveolar Walls, J. Appl. Physiol., 25(6), 689-695.

Fukuoka Y., Kawataa Y., Nikia N., Umetanib K., Nakanoc Y., Ohmatsud H., Moriyamae N., Itohf H. (2014), Microstructure analysis of the secondary pulmonary lobules by 3D synchrotron radiation CT, Medical Imaging: Computer-Aided Diagnosis, edited by Aylward S., Lubomir M. Hadjiiski, Proc. of SPIE Vol. 9035, 1-7.

Fuller B. (1961), Tensegrity. Portfolio and Art News Annual, 4, 112-127.

Greaves I. A., Hildebrandt J., Hoppin Jr. F. G. (2010), Micromechanics of the Lung, Handbook of Physiology - Chapter 14 The Respiratory System, American Physiological Society. 2010.

Henry F. S., Butler J. P, Tsuda A. (2002). Kinematically Irreversible Flow and Aerosol Transport in the Pulmonary Acinus: A Departure from Classical Dispersive Transport, J. Appl. Physiol., 92, 835-845.

Hildebrandt, J., Fukaya, H., and Martin, C. J. (1969) Stress-Strain Relations of Tissue Sheets Undergoing Uniform Two-Dimensional Stretch, J. Appl. Physiol., 27(5), 758-762.

Ingber D. E., Tensegrity I. (2003) Cell structure and hierarchical systems biology. J Cell Sci, $116,1157-1173$.

Kojic M., Tsuda A. (2004). Gravitational deposition of aerosols from oscillatory laminar pipe flows, J. Aerosol Science, 35, 245-261.

Kojic M. and Bathe K. J. (2005), Inelastic Analysis of Solids and Stuctures, Springer.

Kojic M., Vlastelica I., Stojanovic B., Rankovic V., Tsuda A. (2006), Stress integration procedures for a biaxial isotropic material model of biological membranes and for hysteretic models of muscle fibers and surfactant, Int. J. Num. Meth. Engng., 68, 893-909.

Kojic M., Filipovic N., Stojanovic B., Kojic N. (2008), Computer Modeling in Bioengineering, J. Wiley and Sons.

Kojic M., Slavkovic R., Zivkovic M., Grujovic N., Filipovic N. (2010), PAK - Finite Element Program for Srtuctural Analysis, Field Problems and Biomechanics, Faculty of Engineering Sciences, Univ. Kragujevac and Bioeng. Research Center BIOIRC, Kragujevac, Serbia.

Kojic M., Butler J. P., Vlastelica I., Stojanovic B., Rankovic V., Tsuda A. (2011). Geometric hysteresis of alveolated ductal architecture, ASME J. Biomechanics, 133, 1- 11.

Kojic M., Milosevic M., Simic V., Ferrari M. (2014). A 1D pipe finite element with rigid and deformable walls, J. Serb. Soc. Comp. Mech., 8 (2), 38-53.

Liu C. H., Niranjan S. C., Clark J. W. Jr., San K. Y., Zwischenberger J. B., Bidani A. (1998).Airway mechanics, gas exchange, and blood flow in a nonlinear model of the normal human lung, J. Appl. Physiol. 84(4), 1447-1469.

Miki H., Butler J. P., Roger R. A., Lehr J. L. (1993). Geometric Hysteresis in Pulmonary Surfaceto-Volume Ratio during Tidal Breathing, J. Appl. Physiol., 75(4), 1630-1636.

Sasaki, H., and Hoppin, F. G., Jr. (1979), Hysteresis of Contracted Airway Smooth Muscle, J. Appl. Physiol.: Respir. Environ. Exercise Physiol., 47(6),1251-1262.

Tsuda A., Henry F. S., Butler J. P. (1995), Chaotic Mixing of Alveolated Duct Flow in Rhythmically Expanding Pulmonary Acinus, J. Appl. Physiol., 79(3), 1055-1063.

Tsuda A., Filipovic N., Haberthür D., Dickie R., Matsui Y., Stampanoni M., and Schittny J. C. (2008), Finite element 3D reconstruction of the pulmonary acinus imaged by synchrotron Xray tomography, J Appl Physiol., 105, 964-976.

Tsuda A., Laine-Pearson F. E, Hydon P. E. (2011), Why Chaotic Mixing of Particles is Inevitable in the Deep Lung, J. Theor. Biol. 286, 57-66.

Weibel E. R. (2013), It Takes More than Cells to Make a Good Lung, Am J Respir Crit Care Med, 187(4), 342-346.

Weibel E. R. (1963), Morphometry of the human lung, Academic, New York. 
Weibel E. R. and Gil J. (1977), Structure-function relationships at the alveolar level, Bioengineering Aspects of the Lung, New York, 3, 1-81.

Wilson, T. A. (1982), Surface Tension-Surface Area Curves Calculated from Pressure-Volume Loops, J. Appl. Physiol.: Respir. Environ. Exercise. Physiol., 53(6), 1512-1520.

Wilson T. A., and Bachofen H. (1982), A Model for Mechanical Structure of Alveolar Duct, J. Appl. Physiol., Respir. Environ. Excercise Physiol., 52(4), 1064-1070. 